\title{
Metastasizing Pleomorphic Adenoma of the Submandibular Gland with Metastasis to the Lung and Sternum: CT and MR Imaging Findings
}

Tetsuji Yamaguchi, MD1, Kazuto Ashizawa, MD2, Kenji Nagaoki, MD1, Hajime Abiru, MD1, Masataka Uetani, MD1, Tsutomu Tagawa, MD3, Takeshi Nagayasu, MD3, Tomayoshi Hayashi, MD4

${ }_{1}$ Department of Radiology, Nagasaki University School of Medicine, 1-7-1 Sakamoto, Nagasaki 852-8501, Japan.

Tel. No. +81-95-819-7354 Fax No. $+81-95-819-7357$

${ }_{2}$ Clinical Oncology Center, Nagasaki University Hospital

${ }_{3}$ Department of First Surgery, Nagasaki University School of Medicine

${ }_{4}$ Department of Pathology, Nagasaki University Hospital

Short Title: CT and MRI Findings of Metastasizing Pleomorphic Adenoma

Address correspondence and reprint requests to Dr. T. Yamaguchi

Mail address: tetsujiy@net.nagasaki-u.ac.jp 
Key words: Metastasizing pleomorphic adenoma, Submandibular gland, Lung, Bone, CT, MRI

\section{Abstract:}

Distant metastasis of benign pleomorphic adenoma of salivary glands rarely occurs, and these neoplasms have been termed metastasizing pleomorphic adenoma. We present a rare case of metastasizing pleomorphic adenoma of the submandibular gland with lung and bone metastases was obtained MR images and pathological findings. On MRI, the lesions were depicted as an area of high signal intensity on T2-WI, reflecting myxoid or/and chondroid matrices. Metastasizing pleomorphic adenoma should be considered as a differential diagnosis when a pulmonary lesion shows high signal intensity on T2-WI and the patient has a medical history of salivary pleomorphic adenoma. 


\section{Introduction}

Pleomorphic adenomas or benign mixed tumors are the most common tumor of the salivary glands $[1,2]$. The clinical and pathologic features of pleomorphic adenoma are generally benign, but recurrence can result from incomplete surgical excision [1-3]. Distant metastasis of pleomorphic adenoma rarely occurs, and these neoplasms have been termed metastasizing pleomorphic adenoma or metastasizing mixed tumor [1-5]. The clinical and pathological features of metastasizing pleomorphic adenoma have been reported [1,2,5-9], but its imaging findings have rarely been reported. We report a case of metastasizing pleomorphic adenoma of the submandibular gland with lung and bone metastasis.

\section{Case Report}

A 40-year-old woman was admitted with a cough and dyspnea on exercise. Her medical history included a total resection of the right submandibular gland for pleomorphic adenoma seven years ago. Her physical examination and laboratory findings were normal.

The initial chest radiograph showed a mass and a nodule in the left upper and right lower lung zones. A chest CT demonstrated a nodule in the right lower lobe and two lesions in the left upper and lower lobes (Fig. 1A, B). These 
lesions had lobulated shapes with homogeneous soft tissue attenuation. The two lesions in the left upper and lower lobes had broad contact with the left major fissure. A small osteolytic lesion was seen in the body of the sternum (not shown). No swelling of mediastinal lymphnodes was revealed.

On MRI, these pulmonary lesions showed low signal intensity on T1-weighted images (WI) (Spin echo [SE] 902/10) and heterogeneous intermediate to high intensity on T2-WI (Fast SE 7216/90.0) (Fig. 2A). The lesion in the body of the sternum demonstrated low signal intensity on T1-WI and high intensity on T2-WI (Fig. 2B).

A percutaneous needle biopsy with CT guidance was performed for the mass in the left upper lobe. Chondromatous hamartoma or chondroma was pathologically suspected. An apico-posterior segmentectomy of the left upper lobe and partial resection of basal segment of the right lower lobe and the superior segment of the left lower lobe were performed. The all specimens were elastic, hard, and yellowish white in color. They were almost surrounded by normal lung parenchyma, but the mass in the left upper lobe extruded toward the pleural surface.

The microscopic findings of the specimens revealed a large number of epithelial components (Fig. 3A). The tumors were composed of nests and sheets of rounded and cuboidal epithelial cells with eosinophilic or clear cytoplasm 
and intervening myxoid stroma. Chondroid components were also observed (Fig. 3B). The tumors had some necrosis foci, and tumor emboli in the peripheral pulmonary arteries with extension beyond the elastic fibers were revealed by Elastica van Gieson stainning (Fig. 3C). The tumor in the left lower lobe had partial fibrous capsule defects and had invaded into the visceral pleura, but did not extend over the external pleural surface. Mitoses were detected in some tumor cells (Fig. 3D). On average, the number of mitoses was about one or two per 10-high power fields. The pathological diagnosis was pleomorphic adenoma with cellular atypia.

We correlated the pathological findings of the pulmonary lesions with those of the MR imaging to focus on the distribution of the stromal components and epithelial elements. The abundant stromal components that were associated with myxoid or/and chondroid matrices mainly showed a high signal intensity, and the sparse epithelial elements demonstrated intermediate intensity on T2-WI.

The pulmonary lesions were suspected to be metastases from the submandibular tumor because of the patient's medical history. The pathological features of the right submandibular gland tumor, which had been proven to be a pleomorphic adenoma, were similar to those of the pulmonary tumors. Some tumor cell mitoses were also observed. Based on these pathological findings, the pulmonary lesions were diagnosed as distant metastases from the 
pleomorphic adenoma of the submandibular gland; i.e. a metastasizing pleomorphic adenoma.

The patient was followed-up for two years after surgery. However, she developed a cough and back pain and was re-admitted to our hospital. A large mass adjacent to the left pulmonary hilum was revealed on a chest radiograph and CT (Fig. 4A). The osteolytic lesion of the sternum was markedly increased in size with disruption of the bony cortex. On MRI, the left perihilar mass was seen as an area of low signal intensity on T1-WI (SE 923/14) and heterogenous intermediate to high intensity on T2-WI (Fast SE 3000/78) (Fig. 4B). The bone lesion was observed as low signal intensity on T1-WI and high intensity on T2-WI (Fig. 4C). No biopsy or surgical resection was not performed. However, pulmonary recurrence and bone metastasis was strongly suspected because of the MR imaging characteristics of the lesions and the clinical outcome. Conventional extra-beam radiotherapy (45 Gy) and chemotherapy (2400 mg of 5-fluorouracil and $340 \mathrm{mg}$ of nedaplatin) were attempted for the perihilar and bone lesions, but had little therapeutic effect. Surgical resection, a left completion pneumonectomy and resection of the body of the sternum, were carried out after 4 months. The specimens obtained a similar macroscopic appearance to those provided by the previous operation. The perihilar mass was almost completely surrounded by a fibrous capsule with a compressive growth, 
but it extruded toward the pleural surface and invaded into the pericardium. The microscopic findings of the pulmonary and sternal specimens showed pleomorphic adenoma with cellular atypia and were consistent with metastasizing pleomorphic adenoma.

\section{Discussion}

The clinical and pathologic features of pleomorphic adenoma are generally benign, but recurrence can result from incomplete surgical excision [1-3]. Malignant mixed tumors of salivary gland origin have poor prognoses that often involve rapid death despite various therapeutic attempts. The group of malignant mixed tumors includes carcinoma arising in pleomorphic adenomas (so called carcinoma ex-pleomorphic adenoma) and carcinosarcoma (so called true malignant mixed tumor).

Pleomorphic adenoma rarely metastasizes, and there have been only a few reports of its distant metastasis [5-8]. These neoplasms have been termed metastasizing pleomorphic adenoma or metastasizing mixed tumor of the salivary gland [1-3]. Metastatic lesions have been discovered in the bone, lung, lymph nodes, oral cavity, pharynx, skin, liver, retroperitoneum, kidney, calvarium, and central nervous system [5-8]. Metastasizing pleomorphic adenoma is a histologically benign but biologically controversial entity. In 
terms of the pathological features of metastasizing pleomorphic adenoma, malignant components such as anaplasia and increased mitotic activity are not generally seen in primary tumors and metastasizing lesions. However, a few cases demonstrated some cellular pleomorphism as well as the presence of mitoses, which are not sufficient to warrant a diagnosis of malignancy [1, 4]. The mitoses in the reported cases varied from 0-3.4 mitoses per 10 high-power fields, with an average mitotic count of 1 mitosis per 10 high-power fields [1]. In our case, some mitoses and cellular pleomorphism were also observed, but the pathological profile was consistent with metastasizing pleomorphic adenoma.

The imaging features of pleomorphic adenoma of the salivary gland has been reported previously [11,12]. MRI is regarded as especially useful for the imaging of pleomorphic adenoma of the salivary gland. It tends to have a homogeneous or heterogeneous appearance, showing an intermediate to low signal intensity on T1-WI and a high signal intensity on T2-WI. The stromal components associated with myxoid or/and chondroid matrices show high signal intensity, and epithelial elements show intermediate intensity on T2-WI. In contrast studies, the stromal components are demonstrated as marked enhancement and epithelial elements are shown reduced enhancement. 
Only a few case reports have given detailed descriptions of the imaging features of metastasizing pleomorphic adenoma [5]. Because metastasizing lesions have similar pathologic findings to primary tumors, we presume that the imaging findings of metastasizing pleomorphic adenoma are similar to those of salivary gland pleomorphic adenomas. In fact, the pulmonary lesions in our case had a homogeneous appearance with a low signal intensity on T1-WI and a high signal intensity on T2-WI. Based on the correlation between the pathological findings of pulmonary lesions and those of the MR imaging, the abundant stromal components associated with myxoid or/and chondroid matrices mainly showed high signal intensity, and the sparse epithelial elements demonstrated intermediate intensity on T2-WI.

In our case, the pulmonary lesions were suspected to be chondromatous hamartoma or chondroma preoperatively because of a lack of information about medical history of the patient. Pulmonary chondromatous hamartoma, chondroma, and chondrosarcoma are often seen as areas of high signal intensity on T2-WI as well as metastasizing pleomorphic adenoma due to their abundant chondroid matrix [13]. Therefore, differentiation among them is radiologically difficult. It has been reported that a complete and accurate medical history is one of the most helpful elements for differential diagnosis [4]. However, the signal intensity on T2-WI of metastasizing pleomorphic adenoma of the lung 
may be higher than that of primary lung cancer or pulmonary metastasis from common primary tumors (such as colorectal cancer).

The mechanism underlying the metastatic behavior of salivary pleomorphic adenomas is unclear. Because most reported cases occurred after surgical treatment of primary or recurrent lesions of the salivary gland, it has been hypothesized that surgical manipulation may cause tumor cell dislodgement and vascular implantation with subsequent hematogenous spread [5]. An other hypothesis suggests that metastasis to the lung might be due to seeding of aspirated tumor cells [7]. In our case, the former hypothesis is suggested because the pulmonary lesion in the left lower lobe contained some tumor emboli in the peripheral pulmonary arteries.

In conclusion, we have reported a case of metastasizing pleomorphic adenoma, for which we obtained MR images and pathological findings. On MRI, the pulmonary lesions were depicted as an area of high signal intensity with areas of intermediate signal intensity on T2-WI. It was presumed that the abundant stromal components associated with myxoid or/and chondroid matrices of pulmonary lesions demonstrated high signal intensity and the sparse epithelial elements showed intermediate intensity on T2-WI. Metastasizing pleomorphic adenoma should be considered as a differential diagnosis when a pulmonary lesion shows a high signal intensity on T2-WI and the patient has a 
medical history of salivary pleomorphic adenoma. 


\section{References}

1. Wenig RM, Hitchcock CL, Ellis GL, et al. Metastasizing mixed tumor of salivary glands: a clinicopathologic and flow cytometric analysis. Am J Surg Pathol 1992; 16: 845-858

2. Chen KTK. Metastasizing pleomorphic adenoma of the salivary gland. Cancer 1978; 42: 2407-2411

3. Colby TV, Koss MN and Travis WD. Chapter 4 Benign epithelial neoplasms. (In) Atlas of tumor pathology, tumors of the salivary glands. 39-57, 1995, AFIP, Washington D.C.

4. Colby TV, Koss MN and Travis WD. Chapter 5 Malignant epithelial neoplasms. (In) Atlas of tumor pathology, tumors of the salivary glands. 228-251, 1995, AFIP, Washington D.C.

5. Marioni G, Marino F, Stramare R, et al. Benign metastasizing pleomorphic adenoma of the parotid gland: a clinicopathologic puzzle. Head \& Neck 2003; 25: 1071-1076

6. Klijanienko J, El-Naggar AK, Servois V, et al. Clinically aggressive metastasizing pleomorphic adenoma: report of two cases. Head \& Neck 1997; 19: 629-633

7. Wermuth DJ, Mann CH, Odere F. Metastasizing pleomorphic adenoma arising in the soft palate. Otolaryngol Head Neck Surg 1988; 99: 505-508 
8. Chen IH, Tu HY. Pleomorphic adenoma of parotid gland metastasizing to the cervical lymph node. Otolaryngol Head Neck Surg 2000; 122: 455-457

9. Collina G, Eusebi V, Carasoli. Pleomorphic adenoma with lymph-node metastases: report of two cases. Path Res Pract 1989; 184: 188-193

10. Jin $\mathrm{Y}$, Jin C, Arheden K, et al. Unbalanced chromosonal rearrrangements in a metastasizing salivary gland tumor with benign histology. Cancer Genet Cytogenet 1998; 102: 59-64

11. Kinoshita T, Ishii K, Naganuma H, et al. MR imaging findings of parotid tumors with pathologic diagnostic clues. Clin Imaging 2004; 28: 93-101

12. Shah GV. MR imaging of salivary glands. Magn Reson Imaging Clin N Am 2002; 10: 631-662

13. Sakai F, Sone S, Kiyono K, et al. MR of pulmonary hamartoma: pathologic correlation. J Thorac Imaging 1994; 9: 51-55 


\title{
FIGURE LEGENDS
}

\section{Figure 1.}

\begin{abstract}
A, B. CT demonstrates pulmonary lesions in the left upper and lower lobes.
These lesions have homogeneous soft tissue attenuation in contact with the left major fissure. The mass in the left upper lobe partially extrudes toward to the left lower lobe (arrow).
\end{abstract}

\section{Figure 2.}

A. On MRI, the mass is depicted as an area of high signal intensity within areas of intermediated intensity on T2-WI (Fast spin echo [FSE] 7216/90.0).

B. A small bone lesion of the sternum is also demonstrated as a high signal intensity area (arrow).

\section{Figure 3. Microscopic findings of the pulmonary lesions.}
A, B. A large number of epithelial components with nests and sheets of rounded and cuboidal epithelial cells are seen with the intervening myxoid stroma.
Chondroid components are also observed (arrows in B).
C. A tumor embolism in the peripheral pulmonary artery with extension beyond the elastic fibers (arrows) is revealed by Elastica van Gieson stainning.
D. Mitosis is detected in a tumor cell (arrow). 
Figure 4. Follow up study two years after the surgical procedure.

A. A coronal reconstructed postcontrast CT image shows a large mass in the left perihilar region.

B. MR imaging demonstrates the left perihilar mass with heterogenous intermediate to high intensity on T2-WI (FSE 3000/78)

C. The bone lesion is demonstrated as an area of high signal intensity on T2-WI (arrow). 

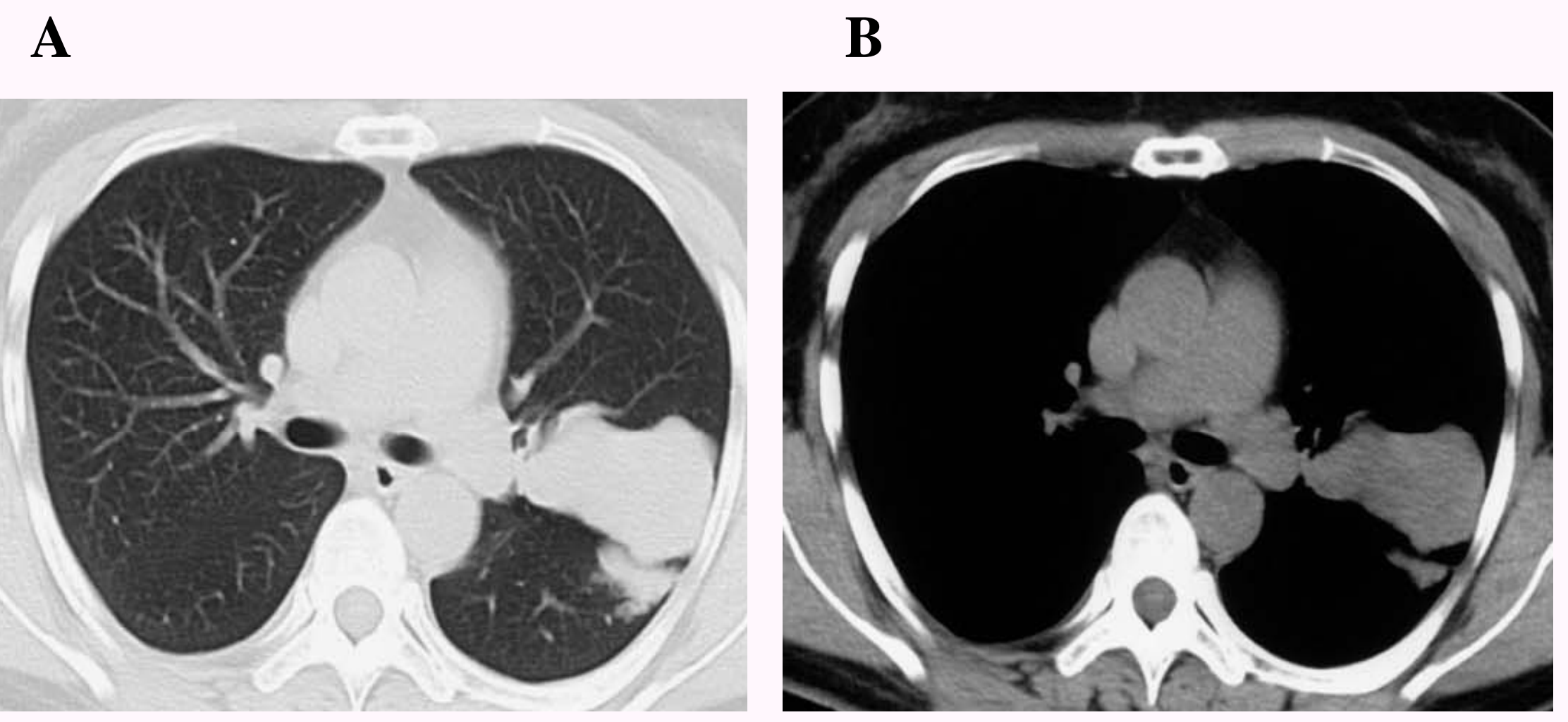

Fig. 1 
A

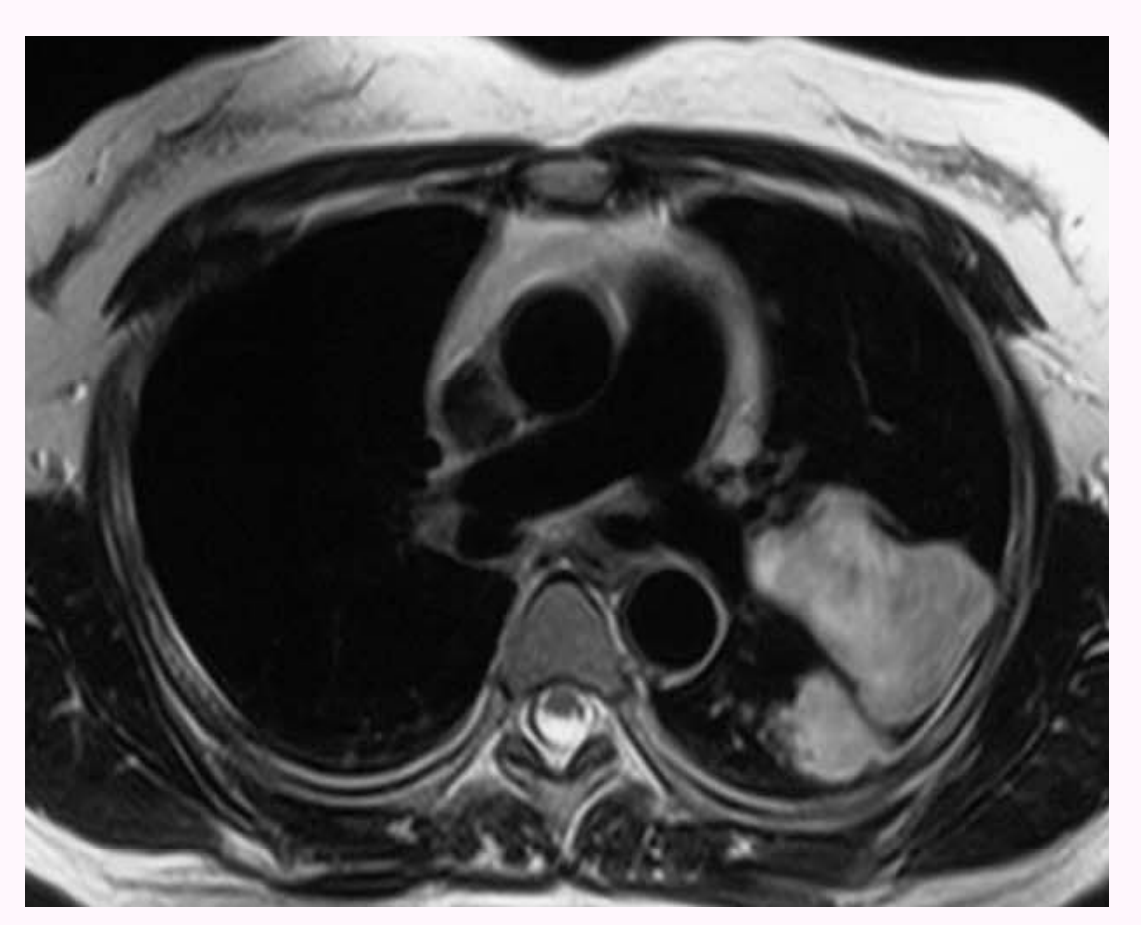

B

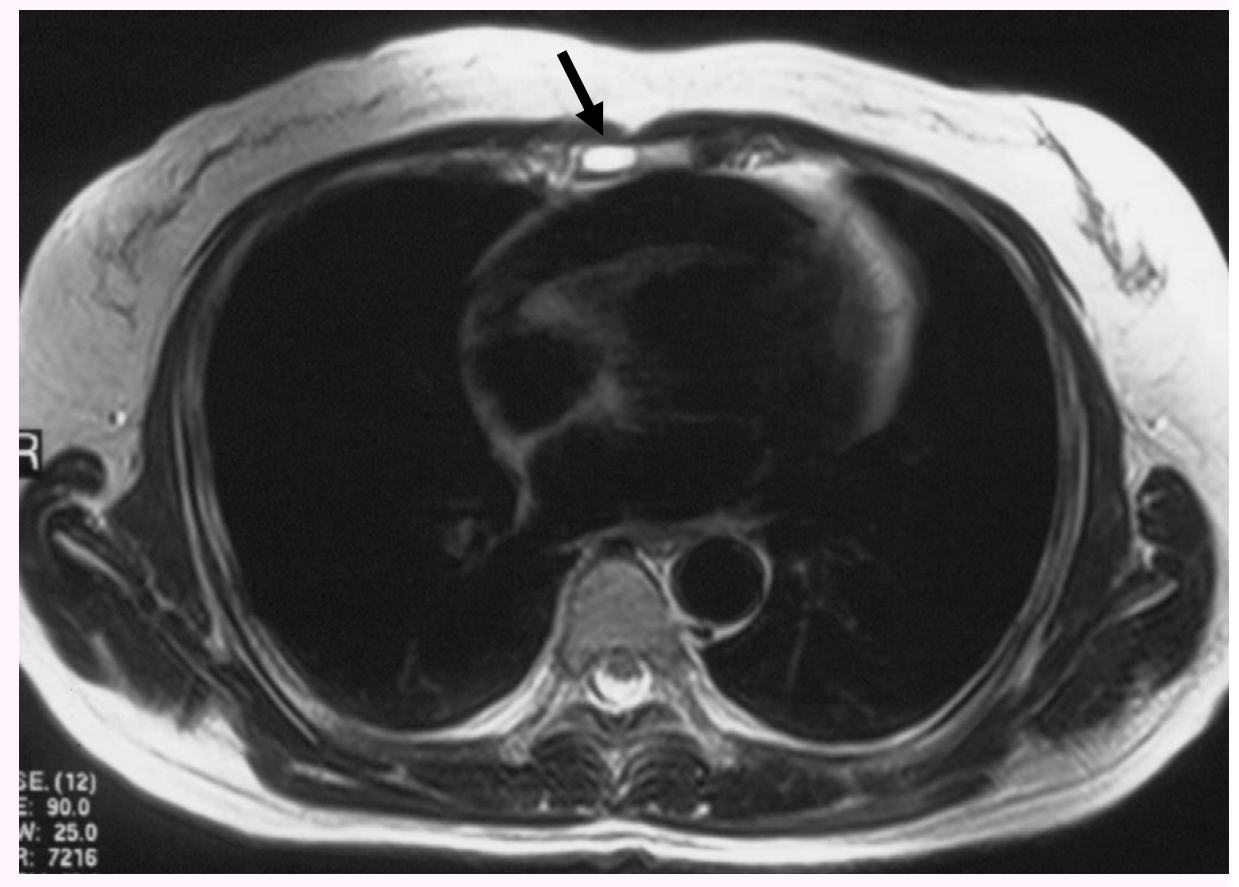

Fig. 2 

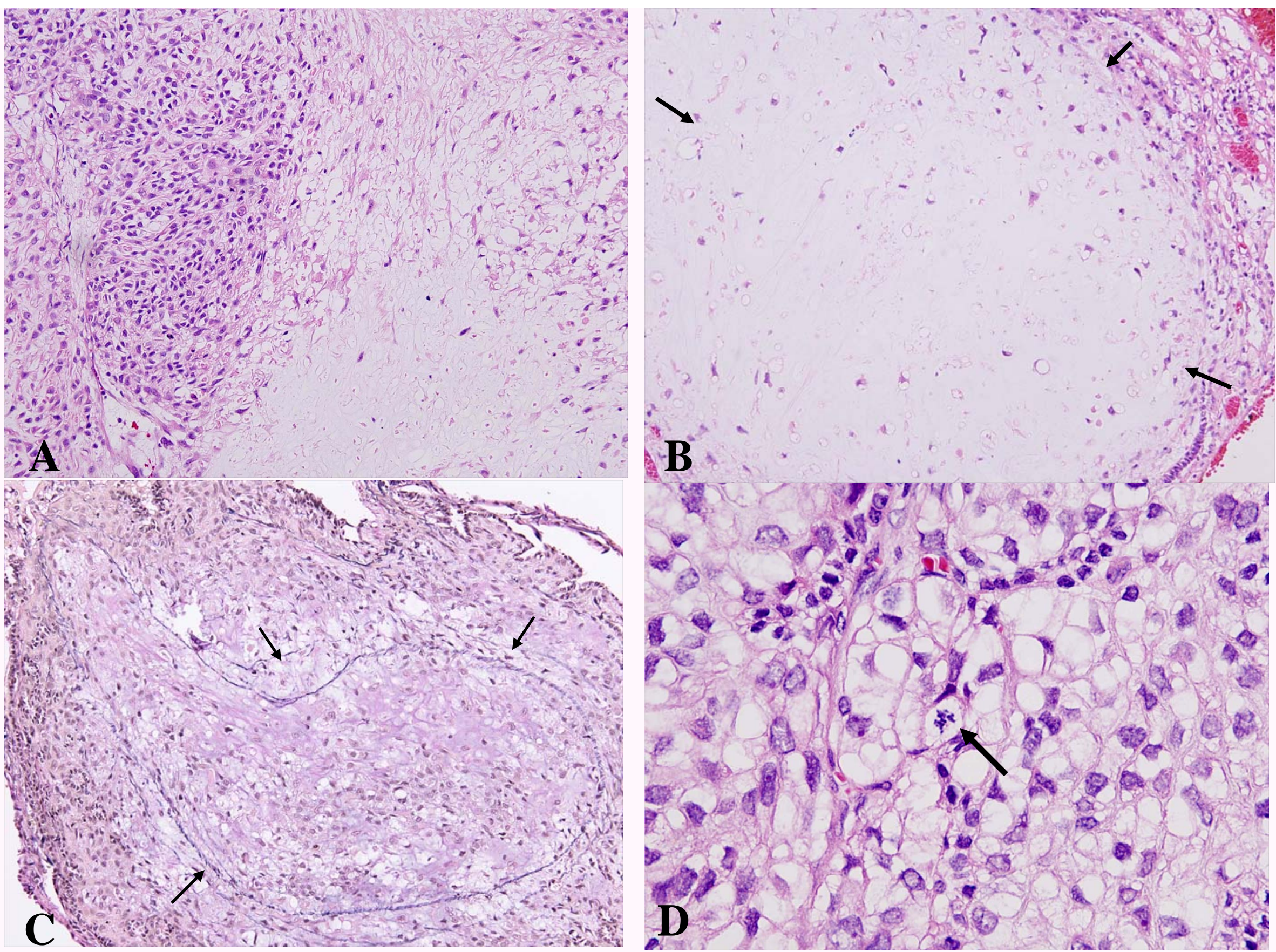

Fig. 3A-D 


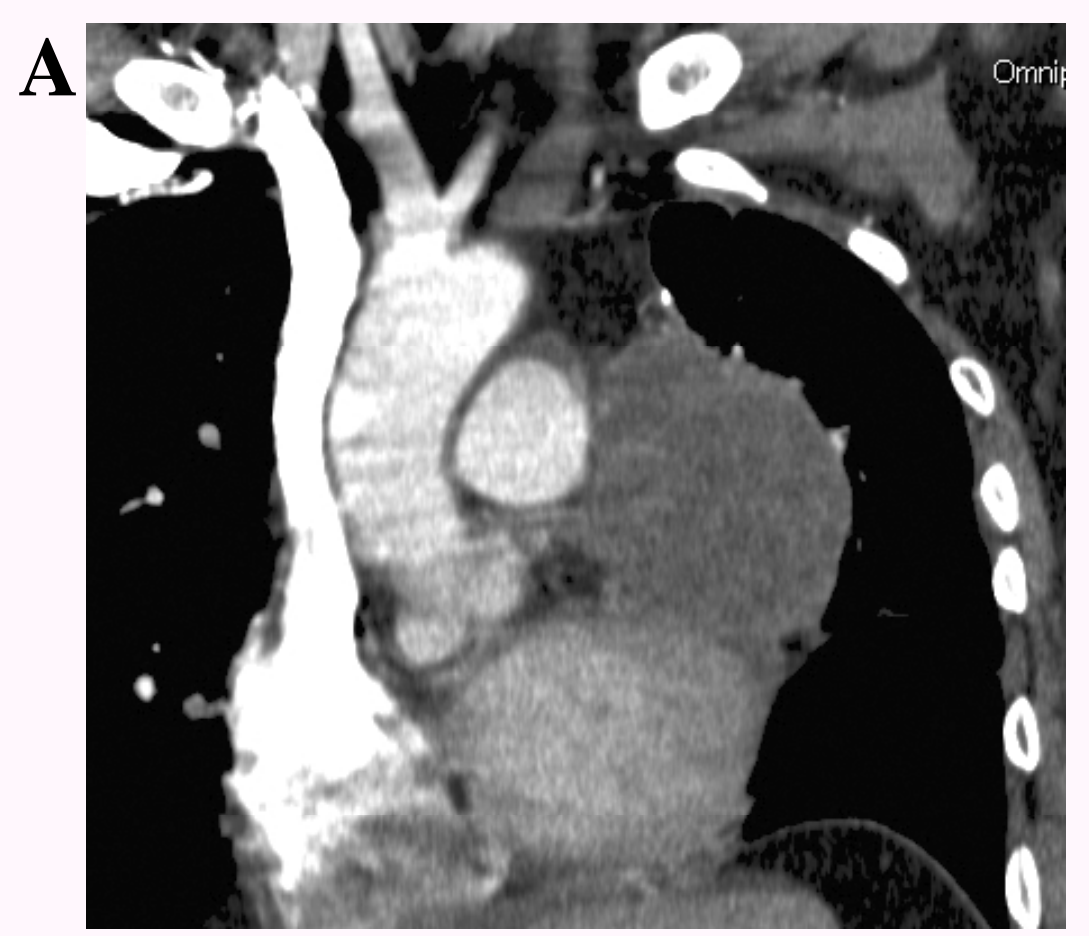

B

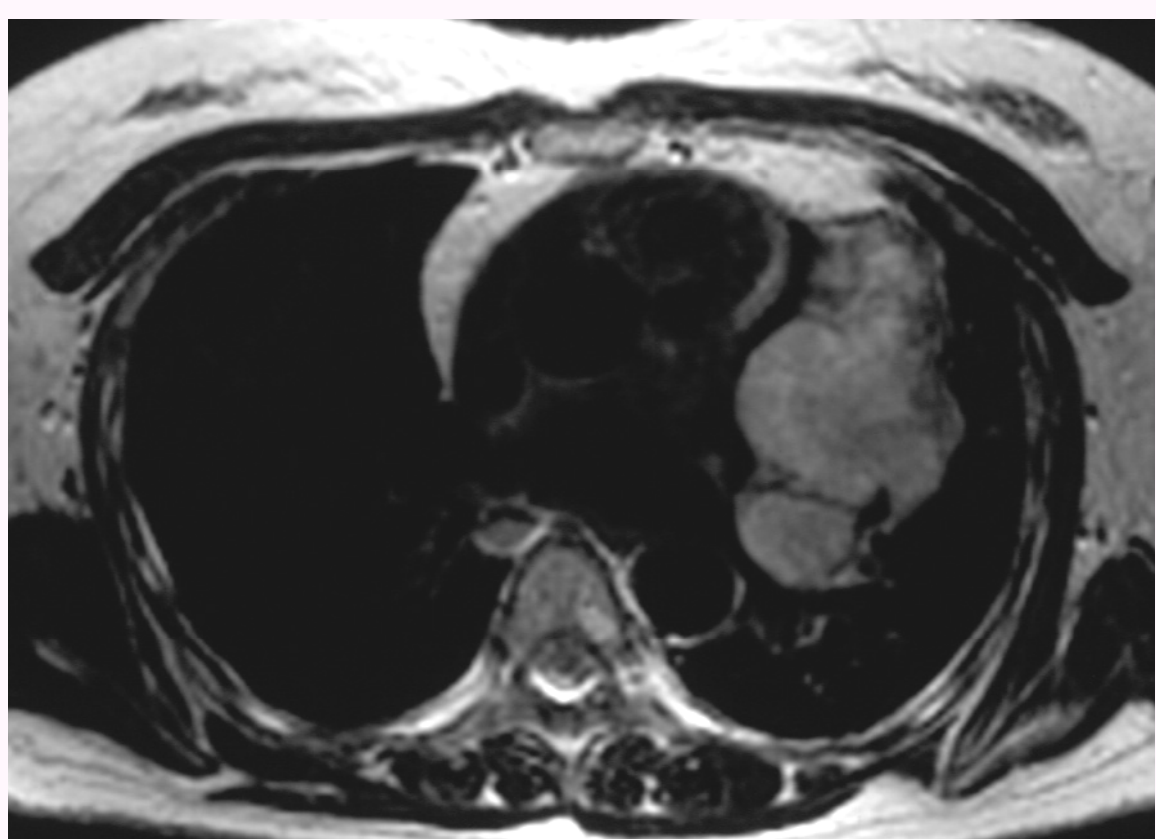

Fig. 4

C

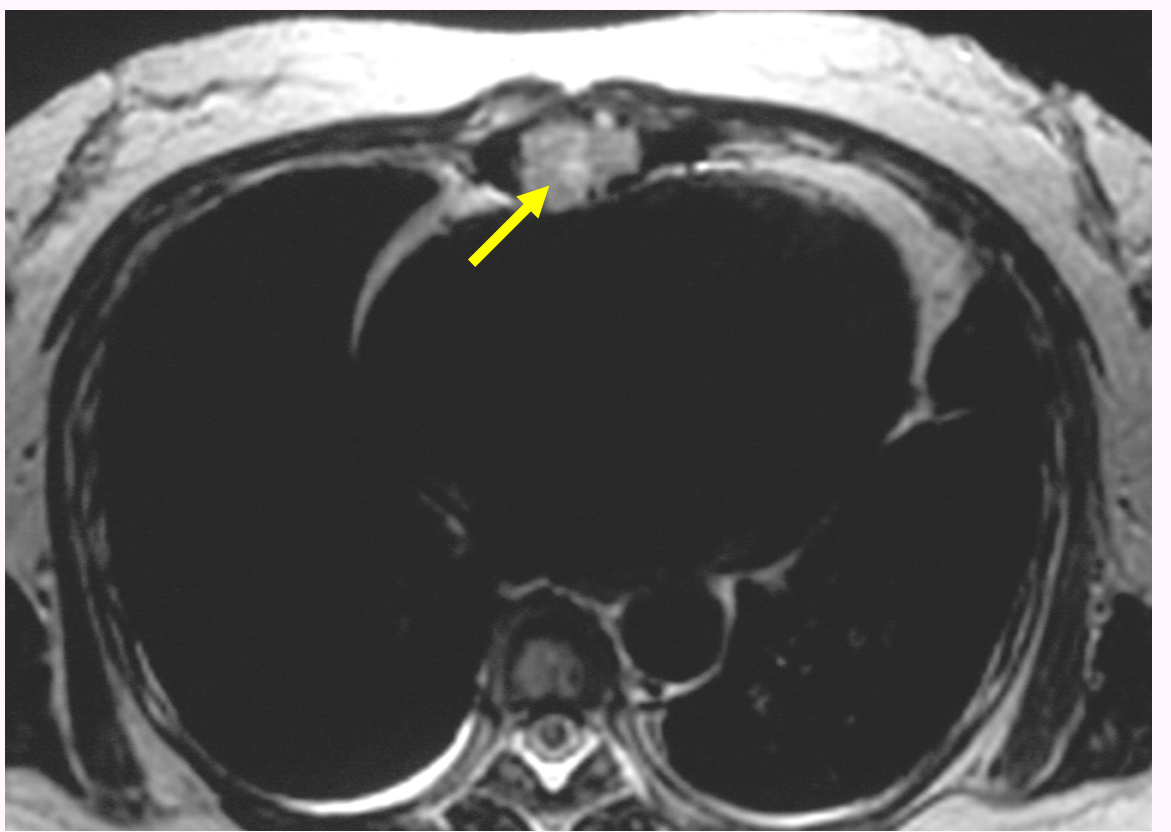

